\title{
Des outils simples en émeri dans le monde égéen
}

Une approche ethnoarchéologique

\section{Athina Boleti}

\section{(2) OpenEdition}

\section{Journals}

Édition électronique

URL : https://journals.openedition.org/artefact/9285

DOI : 10.4000/artefact.9285

ISSN : 2606-9245

Éditeur :

Association Artefact. Techniques histoire et sciences humaines, Presses universitaires du Midi

Édition imprimée

Date de publication : 11 septembre 2014

Pagination : 173-182

ISBN : 978-2-271-08150-6

ISSN : 2273-0753

\section{Référence électronique}

Athina Boleti, « Des outils simples en émeri dans le monde égéen », Artefact [En ligne], 2 | 2014, mis en ligne le 17 mai 2021, consulté le 24 août 2021. URL : http://journals.openedition.org/artefact/9285 ; DOI : https://doi.org/10.4000/artefact.9285

\section{(c) $($ ) $(9)$}

Artefact, Techniques, histoire et sciences humaines est mise à disposition selon les termes de la Licence Creative Commons Attribution - Pas d'Utilisation Commerciale - Pas de Modification 4.0 International. 


\section{Des outils simples en émeri dans le monde égéen. Une approche ethnoarchéologique}

Athina BOLETI ${ }^{1}$

\section{Résumé}

Cet article se propose de contribuer à la discussion concernant la notion de «simplicité» des outils à travers une étude diachronique des outils en émeri dans le monde égéen. Des données ethnoarchéologiques, littéraires et géologiques mettent en évidence le caractère complexe des processus liés à ces outils qui peuvent être qualifiés de simples et viennent ainsi soutenir la thèse que l'absence de technicité au niveau de la mise en forme n'est pas nécessairement équivalente d'une simplicité au niveau de la conception, la matérialisation et l'utilisation de ces outils.

Mots-clés : approvisionnement, Égée, émeri, outils, simple, technicité, utilisation, valorisation.

\section{Abstract}

The aim of the present paper is a contribution to the discussion concerning the concept of "simple» tools through a diachronic study of emery tools in the Aegean. Ethnoarchaeological, literary and geological data show that complex processes are associated to emery tools, which can be characterized as simple. They, therefore, support the view that the absence of technical investment for the shaping of these tools does not necessarily entail simple patterns as far as their conception, realization and use are concerned.

Keywords: Aegean, emery, procurement, simple, technical character, tools, use, valuation. 
La question de la simplicité des outils dans différentes aires chrono-culturelles, de la Préhistoire jusqu'à des périodes plus récentes, a été au cœur de discussions archéologiques et ethnographiques longues et fructueuses ${ }^{2}$. En règle générale, le critère déterminant retenu pour définir ou distinguer un outil simple est l'absence d'investissement technique. Ainsi, un outil dans son expression la plus simple peut être issu d'un support qui n'est pas transformé et qui sert sous sa forme d'origine, brute et naturelle. Toutefois, il a été souligné que, si la forme ou le processus de son obtention sont simples, la simplicité de l'ensemble d'opérations qui le qualifient d'outil reste à discuter. De même, il a été signalé que, dans bon nombre de cas, surtout ethnographiques, ce sont des paramètres socioéconomiques et symboliques qui valorisent l'outil plus que le degré de sa technicité.

L'article qui suit se propose d'aborder la question à travers l'étude de l'exploitation d'une matière minérale qui n'a cessé d'alimenter la panoplie à la fois des artisans spécialisés et des simples paysans en outils simples dans le sens décrit ci-dessus, dans le monde égéen depuis la fin du Néolithique jusqu'à nos jours ${ }^{3}$. Il s'agit de l'émeri, roche métamorphique qu'on trouve dans certaines îles égéennes (notamment Naxos et Paros, mais aussi Samos et Ikaria) et en Asie Mineure (notamment dans le massif du Menderes). C'est une matière minérale très abrasive grâce à la présence de pourcentages de corindon qui peuvent dépasser $50 \%$.

\section{Des outils simples en émeri. Une utilisation diachronique}

L'émeri a servi, entre autres, sous forme d'outils de percussion et d'abrasion, de polissoirs et de pierres à aiguiser. Un témoignage de cette utilisation variée et diachronique est offert par les découvertes faites sur l'île de Naxos, dans les Cyclades, source principale d'émeri en Mer Égée (Fig. 1). On y trouve des outils en émeri dans des contextes archéologiques datés de la fin du Néolithique et de l'âge du Bronze ( $\mathrm{V}^{\mathrm{e}}-\mathrm{II}{ }^{\mathrm{e}}$ millénaires av. J.-C.), mais il faut également noter l'abondance des outils en émeri, notamment des percuteurs, trouvés en surface dans les parties sud et est de l'île. Ce phénomène peut témoigner d'une utili- sation étalée sur différentes périodes du Néolithique jusqu'à nos jours ${ }^{4}$. Par ailleurs, pour ce qui concerne les périodes historiques, des sources littéraires nous apprennent que l'émeri était une matière première particulièrement recherchée et exportée. C'est une pierre à aiguiser réputée pendant l'Antiquité classique si l'on en croit, entre autres, Pindare ( $\mathrm{v}^{\mathrm{e}}$ siècle av. J.-C.) pour qui «la pierre de Naxos qui dompte l'airain et l'aiguise ${ }^{5}$ » se distingue des autres pierres. On n'aurait pas pu voir à Naxos une pierre à laquelle cette description conviendrait mieux que l'émeri. L'émeri est également exploité pendant la colonisation 

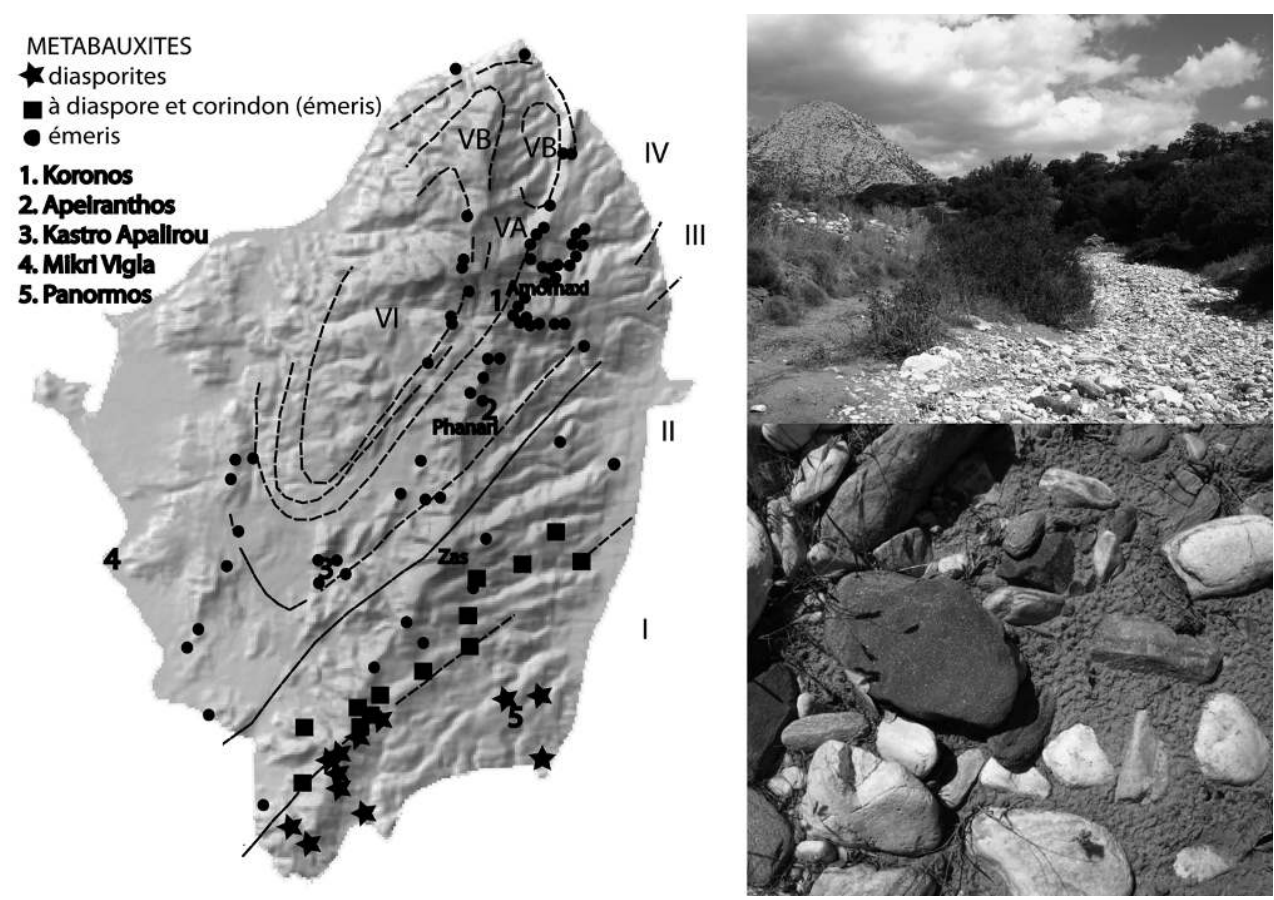

Fig. 1 - a. Carte de Naxos avec les zones de métamorphisme et les principaux gisements de métabauxites

(d'après J. Ben J. Jansen, The geology of Naxos. Geology and geophysical research, Athènes, 1977, et Anne

Feenstra, Metamorphism of bauxites on Naxos, Greece, thèse de doctorat, Université d'Utrecht, Geologica

Ultraiectina, 39, 1985), ainsi que les sites mentionnés dans le texte; $b$. vue de dépôts secondaires d'émeri (lit de torrent) près d'Apaliros, Naxos, 2005, cliché Athina Boleti.

vénitienne, puis l'occupation ottomane de l'île (XIII ${ }^{\mathrm{e}}$-xvIII ${ }^{\mathrm{e}}$ siècles de notre ère). Des sources du xviI siècle nous apprennent que l'émeri est exporté à Smyrne en Asie Mineure, mais aussi en Europe, à Venise et à Marseille ${ }^{6}$.

Enfin, des exemples ethnographiques à Naxos, mais aussi dans l'île voisine de Paros montrent une exploitation récente, voire contemporaine. Selon les témoignages des habitants des villages d'Apeiranthos et de Koronos, dans la région des mines d'émeri à Naxos, des outils en émeri naturel, à savoir des pierres à aiguiser, faisaient partie intégrante de l'équipement des maisons ou des bergeries jusqu'à très récemment. Actuellement, bien que ces outils soient mis hors d'usage ou le plus souvent remplacés par des produits industriels, il est possible de repérer des exemplaires encore utilisés, comme une pierre à aiguiser trouvée dans une ferme abandonnée qui a servi par la suite de lieu de campement, dans la localité Smirigli (le terme signifie émeri en grec) (Fig. 2), ou encore une autre pierre à aiguiser utilisée depuis au moins trois générations par la famille gérant le petit monastère $\mathrm{d}^{\prime}$ Haghia Kyriaki près du village de Lefkes, à Paros?

L'étude d'un échantillon d'outils de percussion et/ou d'abrasion (68) provenant de huit sites égéens (dans les Cyclades et l'île de Crète) de la fin du Néolithique et de l'Âge du Bronze (IVe-II ${ }^{\mathrm{e}}$ millénaires av. J.-C.), mais aussi 

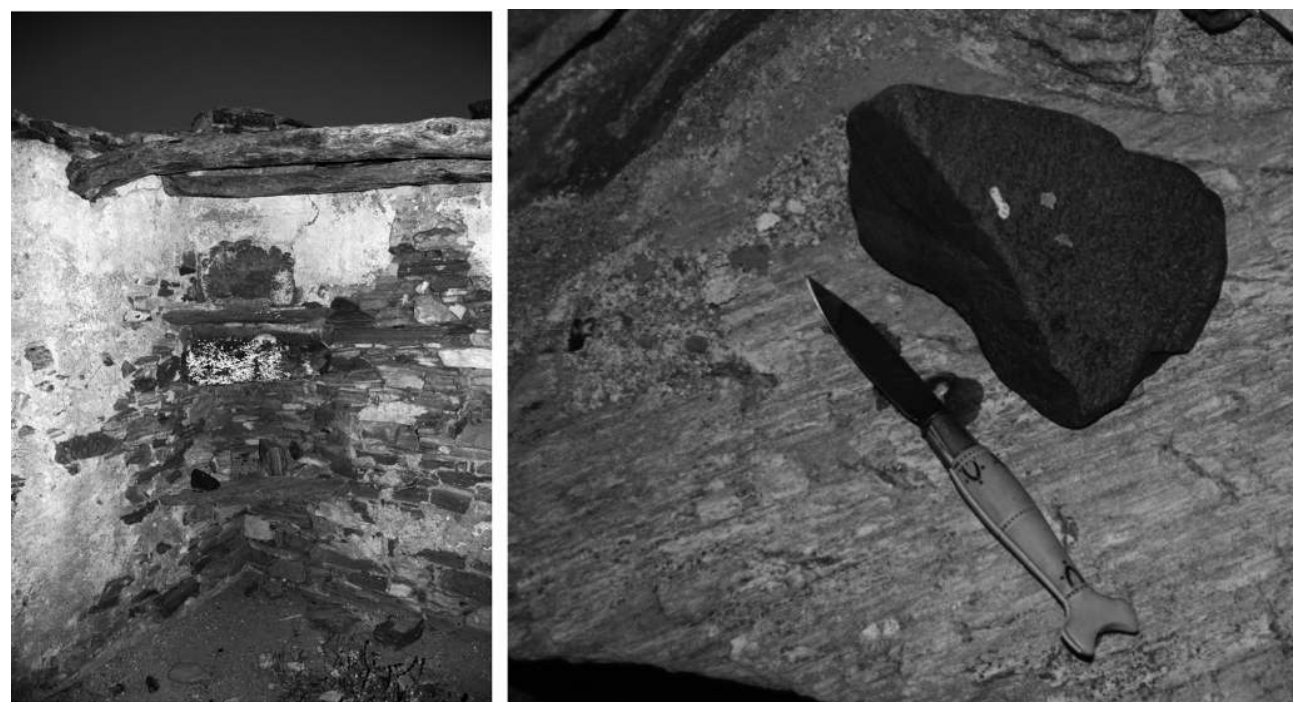

Fig. 2 - Bloc naturel en émeri (échelle: $20 \mathrm{~cm}$ ) utilisé comme pierre à aiguiser dans une ferme abandonnée, localité Smirigli, Paros, 2008, cliché Athina Boleti.

des prospections effectuées dans la partie nord-est de l'île de Naxos ont permis de faire des observations à propos de la simplicité des outils en émeri de la période protohistorique dans le monde égéen.

\section{De la simplicité}

Une analyse techno-morphologique a permis de mettre en évidence l'aspect «simple» de ces outils. L'étude des traces d'usure a montré qu'il s'agit pour la plupart d'outils polyvalents. Seuls six outils portent exclusivement des traces de percussion et dix exclusivement des traces d'abrasion.

Les outils qui portent essentiellement ou majoritairement de traces de percussion (53) ne présentent pas d'indices d'une mise en forme préalable à leur utilisation. C'est donc l'utilisation qui leur confère leur forme résiduelle. Dans certains cas,
Celles-ci peuvent avoir aussi une valeur pour les périodes historiques, puisque le matériel de surface suggère que l'emploi de ces outils a perduré jusqu'aux périodes plus tardives ${ }^{8}$.

de petits aménagements pour la préhension ont été créés par petits enlèvements. Un poli peut aussi être apparu en raison d'une usure tribochimique générée par le contact avec la main lors de l'utilisation. Les formes résiduelles observées sont assez variables (ovale, discoïde, sphérique, cuboïde, ovoïde; de section triangulaire, trapézoïdale) et indiquent, en combinaison avec les dimensions (comprises entre $12,9 \times 9,4 \times 5,4 \mathrm{~cm}$ et $4,7 \times 4,2$ $\times 3,6 \mathrm{~cm}$ ) et le poids (entre 500 et $750 \mathrm{gr}$ ), quel était le support d'origine, à savoir des blocs, des galets et des plaquettes. 

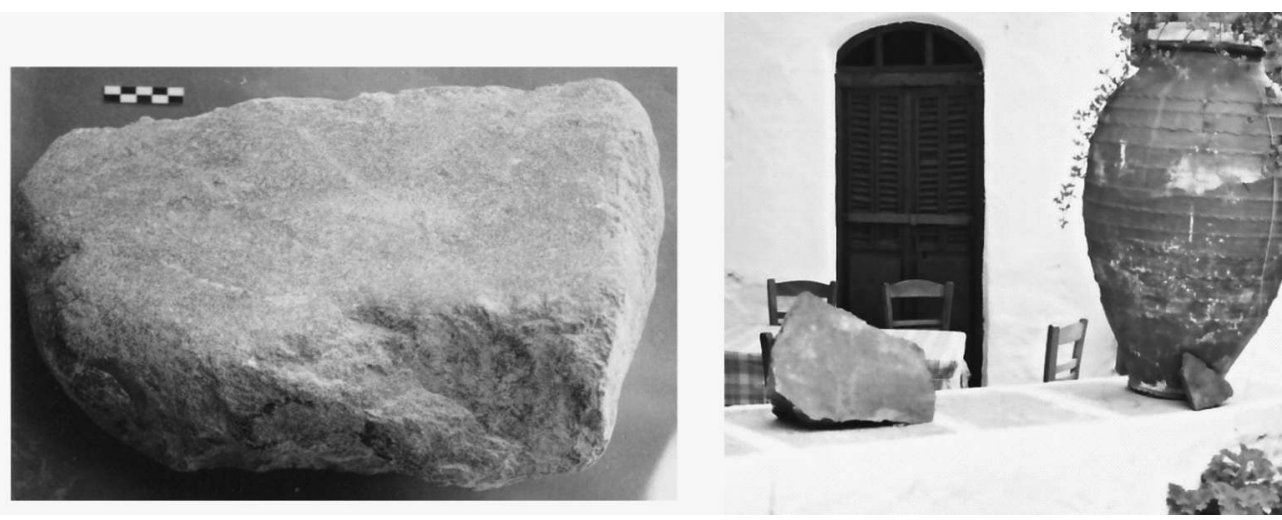

Fig. 3 - a. polissoir en émeri, Akrotiri, Théra, xvi siècle av. J.-C. (échelle: $5 \mathrm{~cm}$ ); b. bloc brut d'émeri avec surface plate et lisse, qui se prête à une utilisation comme pierre à aiguiser, posé sur la place centrale du village de Koronos, Naxos, 2005, clichés Athina Boleti.

Les quinze outils qui portent essentiellement ou majoritairement des traces d'abrasion comprennent aussi bien des pièces passives qu'actives (Fig. 3 et 4 ). Ces pièces sont de formes très variées (sphérique, ovalaire, trapézoïdale, rectangulaire, parallélépipédique, allongée; certains outils de forme particulièrement allongée et de petites dimensions rappellent des tiges ou des barres). Ces formes sont issues de supports naturels, blocs, galets et plaquettes, bien que, dans quelques cas, des traces d'un léger épannelage puissent être identifiées, témoignant d'une mise en forme minime. On peut aussi souvent y voir, comme pour les percuteurs, de petits enlèvements ou des écrasements qui facilitent la préhension.

Les exemples ethnographiques de pierres à aiguiser connus dans les îles de Naxos et de Paros sont pareillement simples. Il s'agit de blocs naturels ayant au moins une surface naturelle plate et lisse (Fig. 2 et 3).

Si la simplicité de ces outils réside surtout dans l'absence d'aménagement, l'approvisionnement en ces supports naturels auprès de gisements peut lui aussi être considéré comme simple. Des études portant sur les gisements et des prospections dans l'île de Naxos ont permis de constater que, pour tous ces outils, les supports naturels peuvent provenir de dépôts secondaires, comme les lits de torrents ou encore les plages de l'île (Fig. 1). L'exploitation de gisements situés près de la surface, tels qu'ils sont connus à Naxos, peut être également envisagée. Là encore, un investissement technique minime aurait été requis. 


\section{De la complexité}

Il y a pourtant une part de complexité dans cette utilisation. D'abord, ce sont les chaînes opératoires et les artisanats dans lesquels ces outils sont impliqués qui sont complexes. Cet aspect de l'utilisation des outils simples au sein des artisanats spécialisés dans le monde égéen a été déjà mis en évidence ${ }^{9}$.

En ce qui concerne l'utilisation des outils en émeri, l'étude des traces d'usure à ce stade de la recherche suggère une grande variabilité et une polyvalence. Bien qu'un référentiel élargi des traces produites par expérimentation soit nécessaire afin de préciser les matières travaillées, on peut envisager le travail des matières dures, comme la pierre. Ainsi, à Naxos, sur les terrasses situées au pied de l'acropole du Bronze ancien à Panormos, la coexistence de percuteurs en émeri retrouvés en surface avec des résidus de taille de blocs de calcaire $^{10}$ suggère une utilisation des percuteurs pour la taille de matériel de construction et cela à différentes époques. À Mikri Vigla, à Naxos, la présence conjointe de fragments d'émeri et de marbre peut être, selon les fouilleurs, un indice d'une activité artisanale comme la fabrication de vases et/ ou de figurines en marbre ${ }^{11}$. Enfin, en Crète, à Mochlos, l'outillage en émeri a été trouvé dans un contexte qui pourrait être associé à la fabrication de vases en pierre selon les fouilleurs ${ }^{12}$.

Des descriptions de bergers de Naxos qui, pendant leur temps libre, travaillent la pierre et la sculptent avec des outils en émeri, ressortent aussi de la poésie naxiote contemporaine, bien que de manière très abstraite ${ }^{13}$.
Le travail du métal (polissoirs et pierres à aiguiser) est l'activité la plus souvent suggérée par la documentation archéologique et littéraire. La difficulté $d$ 'identification des outils en pierre utilisés pour le travail du métal en raison de leur forme peu spécifique et de l'absence d'aménagements a été déjà souligné ${ }^{14}$. Des résidus métalliques sur leurs surfaces sont peut-être l'indice le plus fiable de leur utilisation pour le travail du métal. Bien que de tels cas soient connus dans le monde égéen protohistorique ${ }^{15}$, l'outillage en émeri connu jusqu'ici ne semble pas conserver de traces métalliques. C'est donc surtout le contexte qui permet de l'associer au travail du métal. C'est le cas sur le site d'Akrotiri' ${ }^{16}$, à Théra, et sur celui de $\mathrm{Kommos}^{17}$ ou encore de Cnossos, en Crète. Dans ce dernier, l'hypothèse même d'un atelier travaillant le bronze a pu être formulée pour l'Unexplored Mansion d'où viennent quatre outils en émeri ${ }^{18}$ (Fig. 4). En revanche, les exemples ethnographiques de Paros portent des résidus métalliques très nets, soit sur les arêtes de la surface active soit sur toute la surface, bien qu'il s'agisse d'acier et non pas de bronze (Fig. 2).

Il faut également préciser que l'abrasivité importante de l'émeri fait davantage penser à une utilisation des outils en émeri (polissoirs) pendant les phases de façonnage des objets métalliques (par exemple, après le démoulage pour l'enlèvement des formations superficielles d'oxydes) que pendant les phases de finition. De même, si l'on accepte une utilisation des outils en émeri (pierres à aiguiser) pour l'entretien du tranchant des outils métalliques, il faudrait envisager une utilisation 
qui vise plus à refaçonner le tranchant et son angle qu'à le rendre simplement lisse après utilisation.

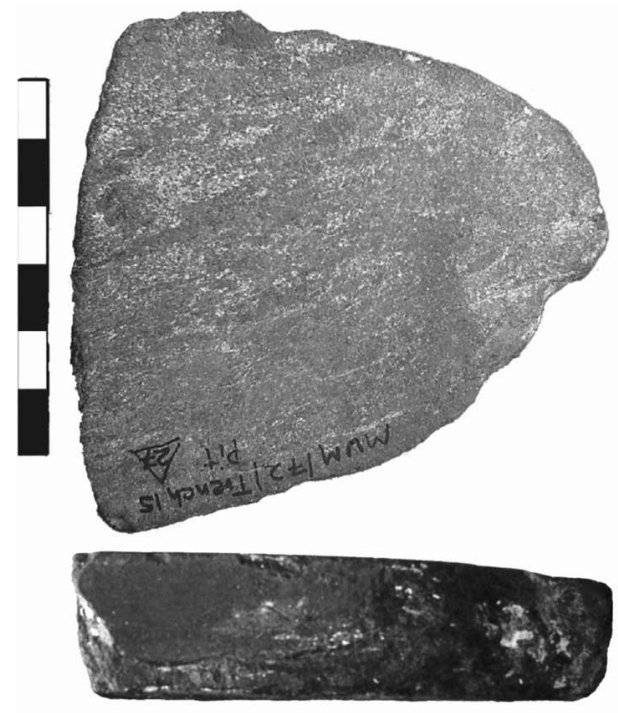

Fig. 4 - Polissoir/pierre à aiguiser en émeri (riche en mica), Cnossos, Unexplored Mansion, $\mathrm{xv}^{\mathrm{e}}$ siècle av. J.-C., cliché Athina Boleti.

Pour résumer, c'est surtout le contexte qui permet de valider les observations faites à partir des traces d'usure et de suggérer que ces outils étaient utilisés dans des artisanats spécialisés comme la construction, la fabrication de vases en pierre et la métallurgie. Ils ont aussi servi très probablement pour l'entretien d'outils métalliques dans toutes les activités artisanales qui en font usage.

Toutefois, pour ce qui concerne les outils en émeri, la complexité réside dans le choix de leur matière première plus que dans leur utilisation. Cette sélection se fait sur deux critères principaux, comme on le remarque généralement dans différents contextes chrono-culturels: la composition minéralogique et la granulométrie ${ }^{19}$. Ces caractéristiques confèrent différentes qualités physiques à la matière première et à l'outil. Deux tendances principales ressortent de l'étude de notre échantillon. Des émeris riches en oxydes de fer et de texture compacte sont utilisés pour les percuteurs. Le poids considérable conféré par les oxydes de fer et la texture compacte favorisent une utilisation en percussion. Les émeris riches en mica et de texture litée sont sélectionnés, respectivement, pour une utilisation en tant que pierres à aiguiser et polissoirs. Cela s'explique si l'on tient compte du fait qu'en s'usant, les couches de mica libèrent des grains de corindon, agent abrasif principal, et permettent ainsi un auto-ravivage de la surface active de l'outil.

À Naxos, le choix se fait au sein d'une gamme d'émeris offrant une variété notable de compositions minéralogiques et granulométriques, puisque l'île constitue un cas unique où l'on trouve tous les degrés de métamorphisme des bauxites en métabauxites, c'est-à-dire en diasporites et en émeris. Plus précisément, sept zones concentriques de métamorphisme d'intensité décroissante ont été distinguées à partir du dôme métamorphique situé dans la partie nordouest du centre de l'île (Fig. 1). Ces zones sont caractérisées par des métabauxites constituées de pourcentages de corindon, de minéraux secondaires et de textures différentes ${ }^{20}$. Au sein du matériel étudié, on a pu, sur des critères macroscopiques, distinguer et attribuer à des zones différentes huit types de métabauxites, parmi lesquels figurent les émeris à corindon et à oxydes de fer à texture compacte et les émeris riches en mica à texture litée. Cette classification a été guidée par des observations issues des recherches géologiques et, pour certains échantillons, par des ana- 
lyses pétrographiques. Bien qu'il soit plus que douteux que les artisans de l'âge du Bronze ou encore les paysans des XIX et $x x^{e}$ siècles de notre ère aient eu conscience que tous ces types appartiennent à la même famille géologique, on ne peut pas nier qu'ils avaient néanmoins une très bonne connaissance de leurs caractéristiques physiques et de leurs propriétés mécaniques, ce qui leur permettait une sélection parfaitement adaptée à leurs besoins en outillage. Il faut donc considérer que des mécanismes cognitifs et des savoir-faire développés permettaient de cartographier cette multitude de paramètres et de les combiner pour arriver au choix de la matière première, en d'autres termes à l'outil.

Un changement radical de la procédure de sélection et de fabrication des outils en émeri (notamment les meules abrasives et les pierres à aiguiser) s'opère pendant l'ère industrielle. Ce sont les gisements d'émeri contenant des pourcentages très élevés en corindon qui sont recherchés au moyen d'études géologiques et minières. La roche est par la suite entièrement broyée, les constituants secondaires, comme les oxydes de fer, éloignés à l'aide de divers procédés et le corindon pur retenu est remoulé avec un liant pour donner de nouveau des outils de formes simples, comme les disques et les plaquettes, et de granulométries homogénéisées variées.

Enfin, est-ce que ces outils simples en émeri sont investis d'une valeur autre qu'utilitaire? Dans l'état actuel de la recherche, il est délicat d'essayer de déceler dans les contextes protohistoriques une valeur symbolique comme celle que lui a attribuée Pindare en disant que l'émeri «dompte» le métal. De même, on peut voir éventuellement une utilisation analogue dans les attestations tardives $\mathrm{du}$ mythe des Aloades et d'Arès. Dans celles-ci, on voit l'émeri sous la mention d'une pierre présente à Naxos, qui, cette fois, «dévore le fer ${ }^{21}$ ». Il est encore plus difficile, voire impossible, de saisir pour les périodes pré- et protohistoriques une valeur plus personnelle et sentimentale, telle qu'elle est dépeinte dans la poésie naxiote contemporaine. Ainsi, des vers $\mathrm{du}$ village d'Apeiranthos nous parlent des Naxiotes qui émigrent et emportent avec eux une petite pierre à aiguiser en émeri pour affûter leur mémoire et ne pas oublier le pays qu' ${ }^{\prime}$ ils quittent ${ }^{22}$.

Toutefois, les études qui s'intéressent à la valeur symbolique attribuée aux outils en pierre préhistoriques, notamment lorsqu'elle est associée à des caractéristiques physiques, comme la couleur, connaissent depuis quelques années un fort élan ${ }^{23}$. À titre d'exemple, des couleurs, comme le noir, le blanc et le rouge sont considérées comme porteuses d'une forte connotation symbolique de caractère très probablement universel ${ }^{24}$. L'émeri présente sans aucun doute des caractéristiques qui pourraient bien générer des associations de cet ordre: tout d'abord la couleur, surtout la couleur noire, caractéristique des variétés riches en oxydes de fer et plus particulièrement en magnétite, puis éventuellement des qualités d'aimant dues à ce même constituant, enfin la dureté ou l'abrasivité, comme on vient de le voir pour des périodes plus récentes ${ }^{25}$. Mais de quels moyens disposons-nous pour mettre en évidence un tel aspect aux périodes pré- et protohistoires? Des interrogations concernant le rôle de la perception et des sens, notamment de la vue et du toucher, non seulement 
dans la valorisation des objets lithiques en général, mais aussi et avant tout dans les processus de leur fabrication, guident les recherches archéologiques les plus récentes ${ }^{26}$. Celles-ci visent à nous livrer, avec l'appui de l'ethnologie et des neurosciences, des clés pour apporter des éléments de réponse plus concrets à cette question.

\section{Épilogue}

En conclusion, les constats qui ressortent de notre cas d'étude viennent s'ajouter à ceux issus d'études précédentes qui montrent que la simplicité, telle qu'elle est définie ci-dessus, n'est pas équivalente à l'absence de technicité dans la conception, la matérialisation ou l'utilisation de l'outil. En revanche, les outils simples en émeri considérés de manière globale et diachronique

\section{Notes}

1. Athina Boleti est ATER à l'Université Paris I Panthéon-Sorbonne. Sa thèse portait sur l'exploitation de l'émeri durant l'Âge du Bronze égéen. Elle a co-publié «The role of tactile perception during stone-polishing in Aegean prehistory ( $5^{\text {th }}-4^{\text {th }}$ millennium B.C.)», WEAR, 271, 2011, p. 2525-2530. Contact: athina.boleti@univ-paris1.fr; http:// www.mae.u-paris10.fr/arscan/Athina-BOLETI. html.

2. Voir Laurence Astruc, François Bon, Vanessa Léa, Pierre-Yves Milcent et Sylvie Philibert (dir.), Normes techniques et pratiques sociales. De la simplicité des outils pré- et protohistoriques. XXVI rencontres internationales d'archéologie et d'histoire d'Antibes, Antibes, APDCA, 2006.

3. Cet article est issu de deux présentations faites en 2007 et 2009 au séminaire sur la question des outils simples organisé par François Sigaut, Sophie A. de Beaune et Hara Procopiou, à l'École des hautes études en sciences sociales; il doit ainsi beaucoup à la réflexion développée au sein de cette équipe. La documentation présentée provient d'une recherche effectuée dans le cadre d'une thèse de doctorat: Athina Boleti, L'exploitation de l'émeri en Égée et en Méditerranée orientale à l'âge du Bronze, Université Paris I Panthéon-Sorbonne, 2009. Le révèlent des mécanismes cognitifs, intellectuels et sensoriels complexes que l'homme a développés en interaction avec son milieu naturel. L'emploi de ces outils est également inscrit dans de structures sociales et économiques elles aussi évoluées, mais moins faciles à déceler, puisque pour le moment la documentation n'est pas conclusive à cet égard.

présent article a également bénéficié de relectures par Hara Procopiou et Françoise Rougemont, que je tiens à remercier ici.

4. Concentrations observées pendant des prospections pour localiser des gisements et dépôts secondaires d'émeris à Naxos dans le cadre du projet ANR «Le forage de la pierre en Méditerranée orientale à l'Âge du Bronze», sous la direction de Hara Procopiou. Une étude spécialisée concernant l'utilisation des percuteurs en émeri pendant les périodes historiques reste à faire. Des expérimentations à cet effet, en collaboration avec Sévérine Moureaud et Hara Procopiou, ont eu lieu en juin 2013 et sont actuellement en cours de traitement.

5. Pindare, $V I^{e}$ Isthmique, 105-108; texte et traduction: Aimé Puech, Pindare. Tome IV. Isthmiques et Fragments, Paris, Les Belles Lettres, 1961.

6. Pour une synthèse détaillée, A. Boleti, 2009, op. cit., p. 44-45, Nikos Sphyroeras, «L'émeri naxien. Contribution à son histoire dès l'Antiquité jusqu'à nos jours», T'Aperathou, $\mathrm{n}^{\circ} 1$ (33-34), mai 1983-février 1986 (en grec).

7. Pour plus de détails, A. Boleti, 2009, op. cit., p. 70-73.

8. A. Boleti, 2009, op. cit., p. 149-171. 
9. Élise Morero et Hara Procopiou, «Des outils simples pour des artisans spécialisés: l'exemple du Quartier Mu de Malia», dans L. Astruc et al. (dir.), 2006, op. cit., p. 383-390, Hara Procopiou, Techniques, sens et émotions. Autour du polissage en Méditerranée orientale durant l'âge du Bronze, habilitation à diriger des recherches, Université Paris I PanthéonSorbonne, 2013, I, p. 51-63, et III, p. 95-100.

10. Observations faites pendant des prospections dans le cadre du projet ANR ForagePierre, voir note 3 .

11. Robin Barber et Olga Hadjianastasiou, "Mikri Vigla: A Bronze Age settlement on Naxos», Annual of the British School at Athens, 84, 1989, p. 62-162 et 136-137.

12. Jeffrey Soles (dir.), Mochlos IA. Period III. Neopalatial settlement on the coast: the artisan's quarter and the farmhouse at Chalinomouri. The sites, Philadelphie, Instap Academic Press, 2003, p. 113-114 et 130. Pour une discussion et un avis contraire, Élise Morero, Artisanat lapidaire en Crète minoenne. Les techniques de fabrication des vases de pierre, thèse de doctorat, Université Paris I Panthéon-Sorbonne, 2009, p. 534-535.

13. Paraskeui Markou, T'arhinoteleioto kladi, 1986, p. 255 (en grec), cité dans Manolis N. Archontakis et Giannoulis G. Giannoulis, Poésie gravée sur la pierre. Mémoire sociale et poétique autour de l'émeri d'Apeiranthos et Koronos, Naxos, Athènes, Atrapos, 2001, p. 439 (en grec).

14. Par exemple, Barbara Régine Armbruster, "L'outillage en pierre du métallurgiste ancien", dans L. Astruc et al. (dir.), 2006, op. cit., p. 321-332.

15. Hara Procopiou, "Chapitre 2. Les outils lithiques», dans Jean-Claude Poursat (dir.), Le quartier $M u V$, vie quotidienne et techniques au Minoen Moyen II (Études crétoises, 34), Athènes, École Française d'Athènes, 2013, p. 43-66 et 197-112, pl. 2.12.14 .

16. Antikleia Moundrea-Agrafioti, "Outillage lithique», et Anna Michailidou, "Trouvailles métalliques», dans Christos Doumas (dir.), Akrotiri, Théra, Maison Ouest, outillage lithique, métallique, divers, Athènes, En Athinais Arhaiologiki Etaireia, 2007, p. 108 et 236 respectivement (en grec).

17. Harriett Blitzer, «Minoan implements and industries», dans Joseph W. Shaw et Maria C. Shaw (dir.), Kommos I. The Kommos eegion and houses of the Minoan town, Part I. The Kommos region. Ecology and Minoan industries, Princeton, Princeton University Press, 1995, p. 501 et 526-532.

18. Hector W. Catling, «The bronzes and metalworking equipement», et Doniert Evely, «The other finds of stone, clay, ivory, faience, lead, etc.», dans Mervyn R. Popham (dir.), The Minoan Unexplored Mansion at Knossos, London, The British School at Athens, 1984, p. 203-221 et 223-259 respectivement.
19. Entre autres pour l'outillage lithique, Danielle Santallier, Vincent Garon, Jean-Luc Gisclon, Éric Jautée et Shasikante Rantsoras, «Les qualités mécaniques des matériaux lithiques utilisés pour la confection de matériel de broyage et de mouture. Réflexions préliminaires», dans Hara Procopiou et René Treuil (dir.), Moudre et broyer. L'identification fonctionnelle de l'outillage de mouture dans la Préhistoire et l'Antiquité. Volume I. Méthodes, Paris, CTHS, 2002, p. 15-28. Pour les outils en pierre destinés au travail du métal, B. Armbruster, 2006, op. cit., p. 323-324: «le métallurgiste choisissait les pierres pour leurs propriétés naturelles spécifiques, physiques et mécaniques, et en fonction des procédés dans lesquels elles étaient impliquées... Les pierres abrasives requièrent des grains de grosseurs différentes selon leur fonction, pierres à poncer ou à polir. »

20. Pour ce phénomène, voir notamment $\mathrm{J}$. Ben H. Jansen, The geology of Naxos. Geology and geophysical research, Athènes, Institute of geological and mining research of Greece, 1977, et Anne Feenstra, Metamorphism of bauxites on Naxos, Greece, thèse de doctorat, Université d'Utrecht, Geologica Ultraiectina, 39, 1985.

21. N. Sphyroeras, 1983, op. cit.

22. Nikos Sphyroeras, Anthologie de poésie apérathite, Athènes, Association d'Apérathou, 1978, p. 142 (en grec), cité aussi dans M. N. Archontakis et G. G. Giannoulis, 2001, op. cit., p. 435.

23. Plus récemment, voir entre autres Andrew Jones et Gavin MacGregor (dir.), Colouring the Past. The significance of colour in archaeological research, Oxford/New York, Bloomsbury Academic, 2002.

24. Chris Scarre, «Epilogue. Colour and materiality in Prehistoric society», dans A. Jones et G. MacGregor (dir.), 2002, op. cit., p. 227-242. Pour un avis contraire qui conteste l'universalité de la couleur, Michel Pastoureau, Dictionnaire des couleurs de notre temps, Paris, C. Bonneton, 1992; Bleu. Histoire d'une couleur, Paris, Le Seuil, 2006; Noir. Histoire d'une couleur, Paris, Le Seuil, 2008.

25. Pour ce qui concerne une autre catégorie d'outillage lithique en émeri, caractéristique de la même période, à savoir les lames polies de haches, herminettes et ciseaux, pour lesquels une telle valeur symbolique aurait pu être suggérée, les quelques exemples recensés au sein d'un ensemble de ce type d'outillage, majoritairement en diasporite, se sont avérés être avant tout des pièces utilitaires. Pour plus de détails, A. Boleti, 2009, op. cit., p. 244-249.

26. Hara Procopiou, Athina Boleti, Roberto Vargiolu et Hassan Zahouani, «The role of tactile perception during stone-polishing in Aegean prehistory $\left(5^{\text {th }}-4^{\text {th }}\right.$ millennium B. C. $)$ ", Wear, $\mathrm{n}^{\circ} 271$, 2011, p. 2525-2530; H. Procopiou, 2013, op. cit. 